\title{
Políticas de financiamiento: Perspectivas y enfoque de las pequeñas empresas del municipio Riohacha ${ }^{1}$
}

\section{Financing policies: perspectives and approach of the small companies of the Riohacha municipality}

DOI: http://dx.doi.org/10.17981/econcuc.38.2.2017.07

Recibido: may 30/2017 Devuelto para revisión: jul 14/17 Aceptado: oct 5/2017

Jorge Luis Mejía Gómez²

\begin{abstract}
Resumen
El objetivo del estudio fue analizar la perspectiva y el enfoque de las políticas de financiamiento de las pequeñas empresas del municipio de Riohacha. Se realizó bajo el paradigma cuantitativo, de tipo descriptivo, diseño no experimental, transaccional de campo. Arrojando como resultado que las pequeñas empresas comerciales utilizan su capital contable para financiarse e invertir en el crecimiento económico del negocio, al tiempo que estudios internos sientan la base para la toma de decisiones, conforme al conocimiento que se obtiene de determinar el costo entre el incremento del capital contable y endeudamiento en sus diferentes presentaciones.
\end{abstract}

Palabras clave: Políticas de financiamiento; costo de capital; pequeñas empresas; crecimiento económico; crecimiento financiero.

\begin{abstract}
The objective of the study was to analyze the perspective and focus of the financing policies of the small enterprises of the municipality of Riohacha. It was carried out under the quantitative paradigm, of a descriptive type, non-experimental design, transactional field. Spreading as a result that small commercial companies use their stockholders' equity to finance themselves and invest in the economic growth of the business, while internal studies form the basis for decision making, according to the knowledge obtained from determining the cost between the increase of stockholders' equity and indebtedness in its different presentations.
\end{abstract}

Key words: Financing policies; capital cost; small enterprises; economic growth; financial growth.

\footnotetext{
${ }^{1}$ Este artículo científico es derivado del proyecto de investigación realizado en la Universidad Libre con sede en Barranquilla, denominado "Políticas de financiamiento empleadas en las pequeñas empresas comerciales del municipio de Riohacha, durante el segundo semestre de 2017”, se enmarca en la línea de investigación Contabilidad y Finanzas, declarada por el Grupo "Tendencias Contables, Económicas y Administrativas - TCEA".

${ }^{2}$ Ingeniero de sistemas y de redes de datos de la Universidad Simón Bolívar, Especialista en Gerencia Financiera de la Universidad Libre, profesional presupuestal del Ministerio de Vivienda, asesor de Proyectos y Financiero de la gobernación del departamento de la Guajira, docente en redes de datos para System Center.
} 
POLÍTICAS DE FINANCIAMIENTO: PERSPECTIVAS Y ENFOQUE DE LAS PEQUEÑAS EMPRESAS DEL MUNICIPIO RIOHACHA Jorge Luis Mejía Gómez

\section{Introducción}

La productividad y competitividad generadas por el proceso de globalización resaltan la necesidad de implementar nuevas modalidades de organización de trabajo, es decir, un nuevo escenario de la economía mundial exige, tanto de los gobiernos como de los empresarios, la formulación de políticas para adaptarse de manera exitosa al mercado competitivo.

En este sentido, las organizaciones conforme a las políticas corporativas, objetivos, metas y plan de negocios, desarrollan procedimientos administrativos para evaluar la situación de la empresa en un momento determinado, mediante el análisis de los resultados de auditorías continuas, que permiten observar variantes en cada tarea, actividad, así como también determinar debilidades, fortalezas, oportunidades y amenazas, que conllevan a observar áreas de mejora así como el correspondiente planteamiento de políticas de mejora de financiamiento, hecho que se constituye en el objeto de estudio de la investigación.

\section{Escenario actual de las pequeñas empresas en el municipio Riohacha}

En Colombia las empresas pueden clasificarse según su tamaño e ingresos en micro, pequeñas, medianas y grandes, dicha clasificación se encuentra reglamentada y normalizada de acuerdo a la Ley 590 (2000), así como por sus modificaciones mediante la Ley 905 (2004) encontrándose categorizadas según se indica en la tabla 1 .

Como se puede observar en la Tabla 1, las variables que permiten establecer el tamaño de la empresa son la cantidad de personal ocupado y los activos totales de la empresa, encontrándose la pequeña empresa oscilando entre 11 y 50 empleados, con activos no superiores a los $368^{\prime} 858.500$ pesos colombianos, destacándose que al considerar estos parámetros en el departamento de La Guajira, según la Cámara de Comercio de la entidad, en el 2016 se tenía conocimiento de 16.213 registradas o renovadas, de las cuales el $2,2 \%$ corresponden a pequeñas empresas como se muestra en la figura 1.

Tabla 1

Definiciones tamaño empresarial

\begin{tabular}{ccc}
\hline Empresa & Número de trabajadores & Activos totales por valor \\
\hline Microempresa & No superior a los diez (10) trabajadores & $\begin{array}{c}\text { Inferior a quinientos (500) SMMLV/ } \\
\text { excluida la vivienda }\end{array}$ \\
Pequeña & Entre once (11) y cincuenta (50) & $\begin{array}{c}\text { Entre quinientos uno (501) y menos de } \\
\text { cinco mil (5.000) SMMLV }\end{array}$ \\
Mediana & Entre cincuenta y uno (51) y doscientos (200) & $\begin{array}{c}\text { Entre cinco mil uno (5.001) a treinta mil } \\
\text { (30.000) SMMLV }\end{array}$ \\
\hline
\end{tabular}

Fuente: Ministerio de Comercio, Industria y Turismo (2016)

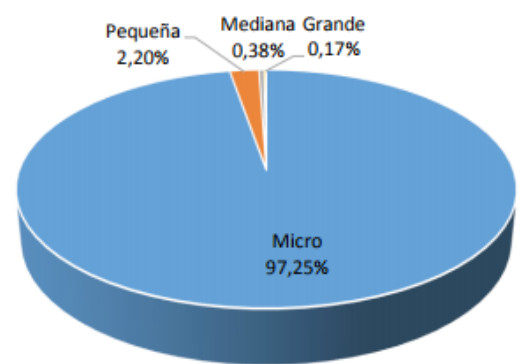

\begin{tabular}{|l|r|}
\hline \multicolumn{1}{|c|}{ TAMAÑO } & CANTIDAD \\
\hline Micro & 15.767 \\
\hline Pequeña & 356 \\
\hline Mediana & 62 \\
\hline Grande & 28 \\
\hline \multicolumn{1}{|c|}{ TOTAL } & $\mathbf{1 6 . 2 1 3}$ \\
\hline
\end{tabular}

Figura 1. Estructura empresarial del Departamento de La Guajira, según tamaño. Fuente: Cámara de Comercio de La Guajira (2017) 
Es de importancia destacar que las pequeñas empresas generan 405.422.186.156 de pesos colombianos (COP \$), poseen el $10 \%$ de la participación en los activos del departamento, aportan 3.131 puestos de trabajo equivalentes al $11,8 \%$ de los empleos de la región, representan el 2,2\% del parque empresarial constituido por un total de 16.213 empresas, siendo los sectores más representativos el comercio al por mayor y al por menor $(41,4 \%)$, construcción (21,3\%), alojamiento y servicios de comida $(10,7 \%)$, otros $(26,6 \%)$; según datos aportados por la Cámara de Comercio de La Guajira (2017).

Sin embargo, aun cuando en Latinoamérica las pymes generan más del $40 \%$ del Producto Interno Bruto (PIB), por lo cual su crecimiento es factor importante para el desarrollo económico, en Colombia, según la revista Dinero (2015), presentan debilidades que tienen como base la falta de innovación, ausencia en el mercado exterior y rentabilidad en el corto plazo para subsistencia entre otros. Estas problemáticas se originan por la dificultad que se presenta en la estructuración y planeación financiera de la empresa a mediano y largo plazo, aspecto avalado por Sierra (2017) por cuanto señala que las pymes colombianas presentan una acentuada inexperiencia en el terreno financiero, lo cual no les permite alcanzar una capacidad logística plena, humana adaptada al tiempo de complejidad y tecnológica de avanzada para brindar competitividad en el mercado internacional.

En este mismo orden de ideas, Salinas (2013) menciona que los problemas de formación de los empresarios Pymes promueven debilidad en la implementación de controles internos, insuficiencia en la calificación del personal para cada una de las diferentes áreas de la empresa, haciendo que una persona atienda diversas actividades para los que no se encuentra adecuadamente capacitado.
De acuerdo a Beltrán (2016), a pesar de que las pymes en Colombia representan el 92\% del total de empresas y que generan el $52 \%$ del total de empleo del país; estas cifras no son respaldadas por su reflejo en el PIB por cuanto sólo generan el $25 \%$ de este y cerca del $25 \%$ de las exportaciones no tradicionales; lo cual evidencia la presencia de dificultades que deben superarse para alcanzar un nivel adecuado de productividad y lograr su supervivencia. Se hace necesario proveer formación sobre fuentes de financiación efectivas, conformación de planes estratégicos, metodologías y herramientas adecuadas para el establecimiento de políticas de costos, pagos y cobros, aprovechamiento de políticas gubernamentales, programas y proyectos enfocados en la exportación, así como la correcta función de la contabilidad en la toma de decisiones.

\section{Vislumbrando las \\ Políticas de Financiamiento}

La gerencia financiera según Ortiz (2005), tiene la responsabilidad de establecer políticas orientadas a garantizar el equilibrio de los objetivos asociados con la liquidez y la rentabilidad, así mismo el manejo adecuado de la liquidez también implica administrar con eficiencia cada área de la empresa. Igualmente, Vargas (2004) las políticas de financiamiento son los lineamientos que le permiten a una entidad obtener fondos 0 recursos financieros para llevar a cabo sus metas de crecimiento y progreso. Por otro lado, Loring, Galán y Montero (2004), consideran que las políticas de financiamiento permiten a las empresas establecer las diversas figuras financieras disponibles para proporcionar a las organizaciones los recursos económicos que en algún momento requieran.

Las políticas financieras para el largo plazo involucran aspectos sobre la inversión, la estructura financiera y la retención y reparto de utilidades de acuerdo a Menguzzato 
y Renau (1991) y por su parte para el corto plazo deben considerar aspectos sobre el capital de trabajo, el financiamiento corriente y la gestión del efectivo.

Dentro de la función financiera se encuentran aspectos fundamentales como los análisis de liquidez y solvencia, de equilibrio económico-financiero, de inversiones, de rentabilidad y beneficios, de fuentes de financiación, de estructura financiera y nivel de endeudamiento, de costos financieros, del riesgo de créditos concedidos como se puede observar en la figura 2. Estos aspectos deben estar alineados con las políticas que direccionan las acciones y decisiones de la empresa a nivel financiero, tal como lo refieren Van Horne y Wachowicz (2010):
Las políticas financieras constituyen el apalancamiento de las empresas, razón por la cual afectan directamente su liquidez, autonomía, solvencia, así como el monto e importancia relativa de los gastos, al igual que la producción. Es por ello, que la relación entre capital propio y crédito invertido en una operación financiera, reducen el capital inicial que es necesario aportar, produciendo un aumento de la rentabilidad obtenida. Según Saldívar (2006), este incremento de apalancamiento aumenta directamente los riesgos de la operación, dado que provoca menor flexibilidad o mayor exposición a la insolvencia o incapacidad de atender los pagos.

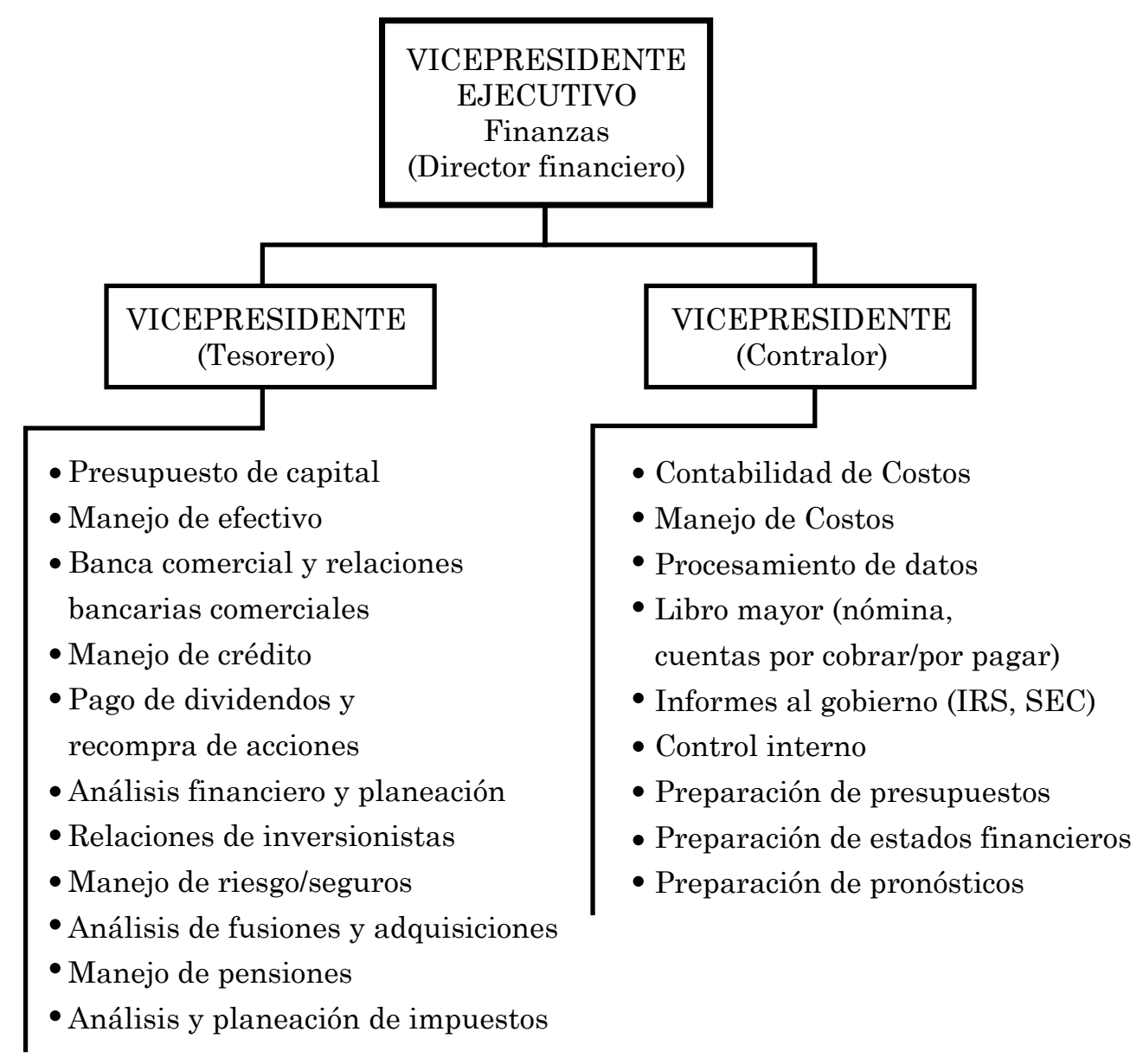

Figura 2. Funciones de la administración financiera Fuente: Van Horne y Wachowicz (2010) 
Por otra parte, Méndez (2006), define las políticas financieras como aquellas que permiten preparar los planes que darán forma a la posición futura de la empresa, tomar decisiones sobre los activos que deben adquirirse, la forma que estos serán financiados y la manera de administrar los recursos con el objeto de obtener una rentabilidad o un beneficio. $\mathrm{Al}$ analizar las definiciones de los autores, vemos como concuerdan en que estas políticas son planes a futuro y se deben proponer a futuro además de ir en concordancia con los objetivos de la empresa u organización.

Igualmente existen otro tipo de políticas que pueden fortalecer el sistema financiero, encontrando entre ellas:

- Políticas de ventas que según Perdomo (2008), comprenden las ventas de contrato riguroso, ventas a crédito sin descuento, ventas a crédito con descuento por pronto pago y ventas de contado, a crédito con y sin descuento, así como la venta al descubierto entendida como una modalidad en la cual el vendedor se compromete a realizar la entrega del bien que aún no obra en su poder.

- Políticas de cobro que para Van Horne y Wachowicz (2010), vienen determinadas por la empresa fijando en ellas sus normas de cobro de cartera mediante la combinación de procedimientos a su vencimiento las cuentas a cargo de clientes, generando equilibrio entre los pagos y el tiempo de recaudo de la cartera.

- Políticas de pago según Améz (2000) comprenden el estudio, evaluación y control eficiente de salidas o desembolsos de efectivo de una empresa, para de esta manera optimizar rendimientos y maximizar el patrimonio o valor de la misma.
Comprendiendo las

Fuentes de Financiamiento

De acuerdo con Ortiz (2005), las fuentes de financiamiento respaldan la operación cotidiana de las empresas, apoyando la ejecución de los planes del negocio, representan un campo complejo de manejo en finanzas. Por su parte, Pérez (2017), establece que la fuente de financiación es la consecución de los medios necesarios para efectuar inversiones, y a cada una de las formas de consecución de estos medios se denomina fuentes financieras:

- Reservas, asumidas como los beneficios obtenidos por la empresa y que no han sido distribuidos entre sus propietarios, atendiendo a Améz (2000), quien señala que las reservas son parte de los beneficios que una empresa genera en un ejercicio económico que no es destinada a dividendo repartible como, consecuencia de que por la ley, por los estatutos o por el acuerdo de la junta general se dedica a dotar las diferentes clases de reservas.

El Plan único de Cuentas para Comerciantes (2013), las definen como aquellos valores que por mandato expreso del máximo órgano social, se han apropiado de las utilidades líquidas de ejercicios anteriores obtenidas por el ente económico, con el objeto de cumplir disposiciones legales, estatutarias o para fines específicos.

- Provisiones, entendidas por el Plan único de Cuentas para Comerciantes (2013), como obligaciones expresas o tácitas a largo plazo, claramente especificadas en cuanto a su naturaleza, pero que, en la fecha de cierre del ejercicio, son indeterminadas en cuanto a su importe exacto o a la fecha en que se producirán. 
Al respeto, Ochoa (2015), refiere que las provisiones no representan hechos acabados, en algunos casos contiene estimaciones para determinar la información que corresponde a cada periodo contable. Constituyen los distintos rubros que poseen las empresas para afrontar imprevistos y estas por lo general son mandato legal.

- Préstamos Bancarios, que de acuerdo a Weston \& Copeland (1999), aparecen en el balance general como documentos por pagar, ocupan el segundo lugar en importancia de crédito comercial como fuente de financiamiento a corto plazo. De igual manera Gitman y Zutter (2012), manifiesta que los Créditos bancarios o cuentas por pagar son la principal fuente de financiamiento no garantizado a corto plazo para empresas comerciales.

- Crédito Comercial, en este aspecto Ortiz (2005) señala que el crédito comercial concedido por los canales de suministro normales no está afectado por tasas de interés, cuando las empresas no cancelan sus compromisos conforme a la fecha convenida, se incurre en un costo de capital causado por los descuentos comerciales perdidos. Por otra parte, Besley (2008), en que esta forma de financiamiento es de utilización rutinaria en las operaciones de cualquier negocio y es una de las más sencillas.

\section{Enfocando al Costo de Capital}

Es el rendimiento requerido sobre los distintos tipos de financiamiento, conocido también como Tasa Mínima Aceptable de Rendimiento (TMAR) para poder calcular su valor, es importante que se tengan en cuenta dos factores fundamentales como son el valor de lo que es la inflación y el premio al riesgo por la correspondiente inversión.
Por su parte, Villareal (1993) indica que sirve como referencia de la rentabilidad mínima que debe obtener la empresa con sus inversiones, ya que la citada renta debe superar el coste de los recursos empleados para financiar la misma, sirve como elemento clave para determinar la estructura financiera de la empresa, o combinación entre las diferentes fuentes financieras y permite relacionar las decisiones de inversión y financiación.

El análisis del costo de capital parte de los factores que lo determinan y, posteriormente, se estudia el costo de cada una de las fuentes financieras que lo forman. Para ello, se agrupan en dos grandes bloques; el coste de los recursos propios y el de los recursos ajenos, finalizando con el costo de capital medio ponderado que sirve como medida del coste de capital.

En cuanto a los costos de los recursos propios, Vélez (2009) establece que son aquellos a los que la empresa no tiene que hacer frente en forma de devolución; por este motivo no tienen un coste explícito, pero si tienen un coste implícito o de oportunidad, que puede calcularse de diversas formas. De acuerdo a lo anterior, constituyen los costos de recursos propios los del capital social y el de la autofinanciación; costos de capital social se presentan cuando una empresa emite acciones, u otros títulos representativos de su capital social, no está obligada a retribuir a sus propietarios con un tipo de interés determinado, mientras que el de la autofinanciación utiliza recursos generados por ella misma como una fuente financiera, por lo que puede parecer que no tienen coste para la empresa. Sin embargo, al igual que ocurre con el capital social, la autofinanciación no tiene un coste explícito, aunque si un coste de oportunidad.

El costo de oportunidad para la Enciclopedia Financiera (2012), es una manera de medir lo que cuesta algo. En lugar de limitarse a la identificación y añadir los costes 
de un proyecto, también identifica la forma mejor alternativa para pasar la misma cantidad de dinero, mientras que el costo de los recursos ajenos son aquellos recibidos por la empresa y que son exigibles, es decir, que se requiere su devolución y no tienen carácter comercial, pudiendo tener varios orígenes según Mascareñas (2001), tales como deuda principal, deuda subordinada, deuda de nominada en moneda extranjera, arrendamientos financieros, entre otros.

\section{Consolidando la Estructura de Capital}

Para Schwartz (1959), consideraba la existencia de una estructura financiera óptima a partir de un uso moderado del apalancamiento financiero, ya que siendo la deuda una forma más barata de financiación se disminuiría el costo promedio de capital y se incrementaría el valor de la empresa. Con el enfoque de la teoría del Trade Off por M\&M (1963), se establece un límite al endeudamiento propuesto a partir de la importancia de los costos de las dificultades financieras y los costos de agencia que contrarrestan los beneficios fiscales de la deuda, además establece que los costos de las dificultades financieras pueden ser de dos clases: directos o indirectos.

Gitman y Zutter (2012), refiere que el capital operativo es aquel que se recupera con la venta de la producción o en el caso de los distribuidores con la venta de los productos comprados a los productores. David (2003) señala que este capital operativo es el principal motivo, por el cual las grandes distribuidoras no necesitan cotizar en bolsa.

En este sentido, el capital operativo de la organización está compuesto por la capacidad de recuperación de la inversión en inventarios que tenga una empresa, producto de las ventas que esta realice y la capacidad de mantener las operaciones en todo momento, mientras que el capital contable es el derecho de los propietarios sobre los activos netos que surgen por aportaciones de los dueños, por transacciones y otros eventos o circunstancias que afectan a una entidad, el cual se ejerce mediante reembolso o distribución. Afirma López (2004), es aquel que se obtiene por la empresa como resultado de la realización de operaciones tales como: reserva legal, utilidades o pérdidas acumuladas, utilidades o pérdidas del ejercicio, efecto monetario acumulado, resultado por tenencia de activos no monetarios entre otros.

\section{Metodológia}

Se realizó un estudio bajo el paradigma cuantitativo, de tipo descriptivo, diseño no experimental, transaccional de campo, para el levantamiento de la información se empleó un cuestionario constituido por 24 ítems, con escala tipo dicotómica para medir la variable. Se determinó la validez del contenido a través del juico de cinco (05) expertos en el área financiera y la confiabilidad mediante el coeficiente de Kuder Richardson (KR-20) para instrumentos dicotómicos arrojó un resultado de 0,91 lo que significa que el cuestionario fue confiable.

Igualmente, a fin de profundizar rasgos inherentes a las políticas empresariales en materia de finanzas, se complementó con una entrevista semiestructurada que permitió ahondar conocimientos en cuanto a estructura financiera, utilidad, estructura de capital, costo de capital y fuentes de financiamiento. Los resultados de la investigación se analizaron utilizando estadísticas descriptivas a través de frecuencias absolutas y relativas, para una muestra aleatoria de sesenta (60) pequeñas empresas de una población de trescientas cincuenta y seis (356) ubicadas en el municipio de Riohacha. 
POLÍTICAS DE FINANCIAMIENTO: PERSPECTIVAS Y ENFOQUE DE LAS PEQUEÑAS EMPRESAS DEL MUNICIPIO RIOHACHA Jorge Luis Mejía Gómez

Tabla 2

Políticas de financiamiento

\begin{tabular}{cccccccc}
\hline & \multicolumn{3}{c}{ Alternativas de respuesta } & \multicolumn{2}{c}{ Total } \\
\cline { 2 - 7 } Indicadores & \multicolumn{3}{c}{$\mathrm{Si}$} & & No & \multicolumn{2}{c}{ Total } \\
\cline { 2 - 7 } & $\mathrm{Fr}$ & $\mathrm{Fa}$ & $\mathrm{Fr}$ & $\mathrm{Fa}$ & $\mathrm{Fr}$ & $\mathrm{Fa}$ \\
\cline { 2 - 7 } & 30 & $50 \%$ & 30 & $50 \%$ & 60 & $100 \%$ \\
Políticas de ventas & 20 & $33 \%$ & 40 & $67 \%$ & 60 & $100 \%$ \\
Políticas de cobro & 44 & $73 \%$ & 16 & $27 \%$ & 60 & $100 \%$ \\
Políticas de pago & 31 & $52 \%$ & 29 & $48 \%$ & 60 & $100 \%$ \\
Promedio & \multicolumn{7}{c}{ Alta } \\
\hline Confiabilidad & \multicolumn{3}{c}{}
\end{tabular}

Fuente: Elaboración Propia (2017)

\section{Resultados}

En el análisis presentado a continuación se hace referencia a una serie de situaciones generadas como resultados, producto de una minuciosa investigación llevada a cabo desde la concepción de la investigación hasta el desarrollo y culminación de la misma, centrada en dos aspectos fundamentales; el análisis y la discusión de los resultados, ello asociado a los objetivos de la investigación. A continuación se presenta un análisis de los resultados obtenidos después de la aplicación de los cuestionarios y entrevistas encuestas realizadas a la población objeto de estudio, iniciando con las políticas de financiamiento reflejados en la Tabla 2.

La tabla 2 engloba los resultados obtenidos para medir la variable "políticas de financiamiento", destacando que los distintos indicadores promedian un 52\% lo cual indica la presencia de atributos en las distintas políticas aunque no de forma representativa ni sustancial, es preciso indicar que mediante las entrevistas semiestructuradas se logró precisar que manejan y establecen procedimientos efectivos para recursos que beneficien el crecimiento del negocio, determinando de manera adecuada los desembolsos de dinero; es decir, aquellos movimientos que le permiten cubrir las obligaciones contraídas y mantener la liquidez necesaria.
En el caso de las políticas de ventas, se equilibra en el 50\% lo cual indica que la mitad de las empresas de la muestra plantean políticas de ventas, mientras que la mitad restante no, se denota la existencia de falencias en el planteamiento de políticas de ventas que a pesar de tener presencia, se encuentra falto de fortalecimiento para que el porcentaje de empresas crezca. A este respecto, Perdomo (2008) expresa que las políticas de ventas comprenden, las ventas de contrato riguroso, ventas a crédito sin descuento, ventas a crédito con descuento por pronto pago y ventas de contado y acredito con y sin descuento; que representan un factor fundamental en toda empresa por cuanto afecta profundamente su capital así como su flujo de dinero.

En cuanto a las políticas de cobro, se evidencia la mayor ausencia de atributos, con un $67 \%$ de negativas, se consolidan como la de menor aplicación por parte de los empresarios, siendo un indicador que perjudica especialmente el flujo, al dificultar el recaudo de las deudas de la empresa. Por último las políticas de pago son el indicador que presenta mayor presencia con una puntuación positiva del 73\%. Según Améz (2000), manifiesta que la política de pago es el proceso que sigue una empresa para determinar de manera adecuada sus desembolsos de dinero. 
Tabla 3

Costo de Capital

\begin{tabular}{cccccccc}
\hline & \multicolumn{3}{c}{ Alternativas de respuesta } & \multicolumn{2}{c}{ Total } \\
\cline { 2 - 8 } Indicadores & \multicolumn{3}{c}{ Si } & \multicolumn{3}{c}{ No } & Fa \\
\cline { 2 - 8 } & Fr & Fa & Fr & Fa & Fr & Fa \\
\hline Costos de los recursos propios & 45 & $75 \%$ & 15 & $25 \%$ & 60 & $100 \%$ \\
Costo de oportunidad & 44 & $73 \%$ & 16 & $27 \%$ & 60 & $100 \%$ \\
Costo de los recursos ajenos & 30 & $50 \%$ & 30 & $50 \%$ & 60 & $100 \%$ \\
Promedio & 40 & $67 \%$ & 20 & $33 \%$ & 60 & $100 \%$ \\
\hline Confiabilidad & \multicolumn{3}{c}{ Alta } \\
\hline
\end{tabular}

Fuente: Elaboración Propia (2017)

En materia de Costo de Capital se obtuvieron los resultados reflejados en la tabla 3. La cual muestra que la variable costo de capital en su indicador Costos de los Recursos Propios arroja que el 75\% de las pequeñas empresas encuestadas utilizan capital propio para autofinanciarse y así no correr riesgos a futuros de endeudamiento, lo que muestra que existe presencia de atributos, a su vez utilizan también, aunque en menor proporción los costos.

De acuerdo a Vélez (2009), los recursos propios no tienen que hacer frente en forma de devolución; por este motivo no representan un coste explícito, pero si tienen un coste implícito o de oportunidad. Es así como constituyen los costos de recursos propios los del capital social y el de la autofinanciación; costos de capital social se presentan cuando una empresa emite acciones, $u$ otros títulos representativos de su capital social, no está obligada a retribuir a sus propietarios con un tipo de interés determinado, mientras que el de la autofinanciación utiliza recursos generados por ella misma como una fuente financiera, por lo que puede parecer que no tienen coste para la empresa.

Sin embargo, al igual que ocurre con el capital social, la autofinanciación no tiene un coste explícito, aunque sin un costo de oportunidad. Para el indicador costo de oportunidad podemos observar que el $73 \%$ de las pequeñas empresas del Municipio de Riohacha encuestadas, prefieren seguir invirtiendo en su negocio ya que es una alternativa económica que le representa un crecimiento en el mismo.

El indicador del costo de los recursos ajenos nos muestra que existe un equilibrio; el 50\% prefiere la financiación de recursos ajenos ya que la empresa es más libre en las decisiones de vinculación del capital a sus activos, tanto en los procesos de expansión como en los procesos de reducción $\mathrm{y}$ el otro $50 \%$ prefiere financiarse con sus propios recursos y así reducir sus costos a futuro.

La tabla 4 muestra los resultados por indicador de la dimensión Estructura Financiera, donde se observa que el capital operativo obtuvo una puntuación del $57 \%$ de los encuestados y coinciden en que las pequeñas empresas del Municipio de Riohacha en estudio manejan un capital de trabajo adecuado, aunque en las entrevistas semiestructuradas refieren que es debido a la utilización como estrategia de manejar reservas económicas y provisiones para el autofinanciamiento, las cuales se van invirtiendo en la empresa casi de forma espontánea a medida que se van generando. 
POLÍTICAS DE FINANCIAMIENTO: PERSPECTIVAS Y ENFOQUE DE LAS PEQUEÑAS EMPRESAS DEL MUNICIPIO RIOHACHA Jorge Luis Mejía Gómez

Tabla 4

Estructura Financiera

\begin{tabular}{|c|c|c|c|c|c|c|}
\hline \multirow{3}{*}{ Indicadores } & \multicolumn{4}{|c|}{ Alternativas de respuesta } & \multicolumn{2}{|c|}{ Total } \\
\hline & \multicolumn{2}{|c|}{$\mathrm{Si}$} & \multicolumn{2}{|c|}{ No } & \multirow[b]{2}{*}{$\mathrm{Fr}$} & \multirow[b]{2}{*}{$\mathrm{Fa}$} \\
\hline & Fr & $\mathrm{Fa}$ & $\mathrm{Fr}$ & $\mathrm{Fa}$ & & \\
\hline Capital accionario & 34 & $57 \%$ & 26 & $43 \%$ & 60 & $100 \%$ \\
\hline Capital contable & 45 & $75 \%$ & 15 & $25 \%$ & 60 & $100 \%$ \\
\hline Promedio & 40 & $67 \%$ & 20 & $33 \%$ & 60 & $100 \%$ \\
\hline Confiabilidad & \multicolumn{6}{|c|}{ Alta } \\
\hline
\end{tabular}

Fuente: Elaboración Propia (2017)

Tabla 5

Fuentes de financiamiento

\begin{tabular}{|c|c|c|c|c|c|c|}
\hline \multirow{3}{*}{ Indicadores } & \multicolumn{4}{|c|}{ Alternativas de respuesta } & \multicolumn{2}{|c|}{ Total } \\
\hline & \multicolumn{2}{|c|}{$\mathrm{Si}$} & \multicolumn{2}{|c|}{ No } & \multirow[b]{2}{*}{$\mathrm{Fr}$} & \multirow[b]{2}{*}{$\mathrm{Fa}$} \\
\hline & $\mathrm{Fr}$ & $\mathrm{Fa}$ & $\mathrm{Fr}$ & $\mathrm{Fa}$ & & \\
\hline Autofinanciamiento por Reservas & 30 & $50 \%$ & 30 & $50 \%$ & \multirow{5}{*}{$\begin{array}{l}60 \\
60 \\
60 \\
60\end{array}$} & \multirow{5}{*}{$\begin{array}{l}100 \% \\
100 \% \\
100 \% \\
100 \%\end{array}$} \\
\hline Autofinanciamiento por Provisiones & 17 & $28 \%$ & 40 & $72 \%$ & & \\
\hline Préstamos Bancarios & 32 & $53 \%$ & 28 & $47 \%$ & & \\
\hline Crédito Comercial & 47 & $78 \%$ & 13 & $22 \%$ & & \\
\hline Promedio & 32 & $53 \%$ & 28 & $47 \%$ & & \\
\hline Confiabilidad & \multicolumn{6}{|c|}{ Media } \\
\hline
\end{tabular}

Fuente: Elaboración Propia (2017)

El indicador de capital contable muestra que el $75 \%$ de las pequeñas empresas del municipio de Riohacha, utilizan su capital contable para financiarse e invertir en crecimiento económico y financiero de su negocio. A su vez, y de acuerdo a las entrevistas, consideran conforme a la experiencia ordinaria de negocio que genera menor costo incrementar el patrimonio en comparación con el endeudamiento.

En cuanto a las Fuentes de Financiamiento, la tabla 5 muestra que el indicador Autofinanciamiento por reservas obtuvo un porcentaje de respuestas equivalente al $50 \%$, lo que significa que la mitad de las pequeñas empresas del Municipio de Riohacha manejan reservas económicas como estrategia para así poder cubrir situaciones de pérdidas en la empresa, en este sentido se observa que no es concluyente por cuanto existe un equilibrio entre las empresas que lo utilizan pero en el mismo porcentaje empresas que lo desconocen o no lo aplican.

En relación con el autofinanciamiento por provisiones, se observa que un $72 \%$ de los encuestados opina que las pequeñas empresas no establecen como fuente de financiamiento las provisiones dentro de sus empresas, así mismo no poseen políticas adecuadas para cubrir el valor de las estimaciones que debe efectuar la empresa.

También se observa que los encuestados consideran que las pequeñas empresas en estudio utilizan como fuente de financiamiento en crédito bancario, este indicador muestra que el 53\% utiliza este tipo de soluciones financieras, es decir, las pequeñas empresas 
buscan usar el dinero para adquisición de inventarios destacándose como uno de los atributos objeto de estudio. Con respecto al crédito comercial, un $78 \%$ de las empresas encuestadas establecen que este tipo de financiamiento se utiliza frecuentemente, destacando en las entrevistas semiestructuradas que ello obedece a que esto representa una forma continua de crédito, donde se evalúan los costos por la adquisición de esta de modalidad de financiamiento, es decir, existe presencia de este atributo.

\section{Conclusiones}

Una vez realizado el análisis sobre las perspectivas y el enfoque que dan las pequeñas empresas del municipio Riohacha a las políticas financieras, se constata que las políticas de pago prevalecen como la política mayormente implementada, en tanto que las políticas de cobro, cobro de cartera y ventas no son utilizadas lo cual genera serias dificultades en las finanzas corporativas.

En lo que respecta a políticas de pago, las pequeñas empresas manejan y establecen procedimientos efectivos para garantizar que los recursos que benefician el crecimiento del negocio estén disponibles en el momento que las necesidades reales de las empresas lo requieran, utilizando en consecuencia una adecuada administración de políticas de pago, sin reflejarlo en sus políticas financieras.

Por otra parte, el autofinanciamiento se sustenta en reservas y provisiones, lo cual evidencia que no se poseen políticas adecuadas para cubrir el valor de las estimaciones que debe efectuar la empresa. Igualmente, destaca el hecho que prevalece la utilización como fuente de financiamiento del crédito bancario así como el crédito comercial debido a las características de su fácil acceso.

Adicionalmente, este tipo de empresas en el contexto estudiado se enfocan en utilizar capital propio para autofinanciarse, evitando asi el riesgo de futuros endeudamientos, bajo la convicción que la inversión estará plenamente compensada con la rentabilidad que se recibirá.

En concordancia, en cuanto al costo de oportunidad las empresas prefieren invertir en el mismo negocio, por cuanto es percibido como una alternativa económica que le representa un crecimiento directo, mientras el indicador de costo de los recursos ajenos evidencia un equilibrio entre la vinculación del capital a sus activos y el financiamiento con recursos propios.

El capital accionario tiende a orientarse hacia el capital de trabajo adecuado por cuanto es de suma importancia debido a la influencia que ejerce en sus operaciones diarias, un activo circulante insuficiente o una mala administración de los pasivos circulantes es una de las causas más comunes del fracaso de toda empresa.

Finalmente, las deficiencias en las perspectivas y enfoques que se dan a las políticas, crea un entorno ineficiente donde se dificulta el funcionamiento de todo el proceso de financiamiento.

\section{Recomendaciones}

- Capacitar a la gerencia de pequeñas empresas en la identificación de factores claves de éxito.

- Desarrollar competencias en el gerente financiero de pequeñas empresas en cuanto al uso adecuado de las fuentes de financiamiento, el proceso de evaluación, manejo de datos fiables y seguros para el análisis y toma de decisiones.

- En cuanto a las políticas de inversión, deben establecerse procesos formales administrativos que permitan cubrir obligaciones contraídas y mantener la liquidez necesaria. 
POLÍTICAS DE FINANCIAMIENTO: PERSPECTIVAS Y ENFOQUE DE LAS PEQUEÑAS EMPRESAS DEL MUNICIPIO RIOHACHA Jorge Luis Mejía Gómez

\section{Referencias}

Améz, F. (2000). Diccionario de contabilidad y finanzas. Madrid: Cultural S.A.

Beltrán, A. (2016). Los 20 problemas de la pequeña y mediana empresa. Bogotá, D.C.: Universidad Externado de Colombia.

Besley. (2008). Fundamentos de Administración financiera. México, D.F.: Cengage learning.

Cámara de Comercio de La Guajira. (2017). Informe actividad empresarial de La Guajira 2017. Riohacha: Cámara de Comercio de La Guajira.

Enciclopedia Financiera. (2012). Enciclopedia Financiera. Recuperado de http:// www.enciclopediafinanciera.com/

Gitman, L., y Zutter, C. (2012). Principios de administración financiera $\left(12^{\circ}\right.$ ed.). México, D.F.: Pearson.

Loring, J., Galán, F. y Montero, T. (2004). La gestión financiera. Bilbao: Deusto.

Mascareñas, J. (2001). El coste del capital. Madrid: Universidad Complutense de Madrid.

Méndez, C. (2006). Diseño y desarrollo del proceso de investigación con énfasis en ciencias empresariales. México, D.F.: Limusa.

Menguzzato, M. y Renau, J. J. (1991). La Dirección Estratégica de la Empresa. Un enfoque innovador del management. Barcelona: Ariel.

República de Colombia. Ministerio de Comercio, Industria y Turismo. (25 de Agosto de 2016). Definición Tamaño Empresarial Micro, Pequeña, Mediana o Grande. Obtenido de http://www.mincit.gov.co/mipymes/publicaciones/2761/ definicion_tamano_empresarial_micro_pequena_mediana_o_grande

Ochoa, J. (2015). Modelos Gerenciales. Un marco conceptual. Medellín: Fundación Universitaria María Cano.
Ortiz, A. (2005). Gerencia financiera y diagnóstico estratégico ( ${ }^{\circ}$ ed.). Bogotá, D.C.: McGraw-Hill.

Perdomo, A. (2008). Elementos básicos de administraciòn financiera $\left(10^{\circ}\right.$ ed.). México, D.F.: Cengage Learning.

Pérez, E. (2017). Curso de Economía de la Empresa. Introducción. Madrid: Centro de Estudios Ramon Areces S.A.

República de Colombia. Congreso de la Republica. (10 de julio de 2000). Por la cual se dictan disposiciones para promover el desarrollo de las micro, pequeñas y medianas empresa. [Ley 590 de 2000]. Bogotá D.C.: DO: 44078.

República de Colombia. Congreso de la Republica. (2 de agosto de 2004). Por medio de la cual se modifica la Ley 590 de 2000 sobre promoción del desarrollo de la micro, pequeña y mediana empresa colombiana y se dictan otras disposiciones. [Ley 905 de 2004]. Bogotá D.C.: DO: 45628.

Saldívar, A. (2006). Planeación financiera de la empresa. Barcelona: Trillas.

Salinas, J. F. (2013). Problemática que afrontan las pequeñas y medianas empresas en Colombia por falta de un sistema de control. Bogotá, D.C.: Universidad Militar Nueva Granada.

Sierra, J. F. (10 de Mayo de 2017). Los problemas que agobian a las pequeñas y medianas empresas. El Colombiano.com [en línea]. Recuperado de http://www. elcolombiano.com/negocios/empresas/capital-y-mano-de-obra-agobian-a-pymespaisas-IJ6496684

Van Horne, J., y Wachowicz, J. (2010). Fundamentos de administración financiera (13ºd. ed.). México, D.C.: Prentice Hall.

Villareal, A. (1993). Evaluacion financiera de proyectos de inversion ( $9^{\circ}$ ed.). Bogotá, D.C.: Norma.

Weston, F. y Copeland, T. (1999). Finanzas en Administración (5 ed.). México, D.F.: Mc Graw Hill. 